\title{
RESENHA
}

\section{EDUCACIÓN EN RAPA NUI: SOCIEDAD Y ESCOLARIZACIÓN EN ISLA DE PASCUA (1914-2014)}

DOI: http://dx.doi.org/10.1590/2236-3459/65319

\begin{abstract}
EDUCATION IN RAPA NUI: SOCIETY AND SCHOOLING IN EASTER ISLAND (1914- 2014)
\end{abstract}

\author{
Andrés Felipe Bianchetti Saavedra \\ Universidad San Sebastián, Chile.
}

\section{$\cos 80$}

CORVALÁN RODRIGUEZ, Javier. Educación en Rapa Nui: sociedad y escolarización en Isla de Pascua (1914-2014). Santiago de Chile: Universidad Alberto Hurtado, 2015.

U

n siglo de desarrollo educativo en Isla de Pascua es el que describe y analiza el antropólogo y doctor en Sociología por la Universidad Católica de Lovaina Javier Corvalán Rodríguez en su libro Educación en Rapa Nui: sociedad y escolarización en Isla de Pascua (1914-2014) editado y publicado por la Universidad Alberto Hurtado en Santiago de Chile en diciembre del año 2015.

El texto se estructura en tres partes diferenciadas en una línea de tiempo concadenada. La primera parte, titulada Educación misional, escuela colonial y escuela estatal religiosa en Rapa Nui, da cuenta de los primeros episodios que pueden ser considerados procesos educativos en la isla. Corvalán señala en este sentido que los encuentros iniciales entre expedicionarios españoles, ingleses y franceses podrían considerarse momentos de educación informal en Rapa Nui, donde existió aculturación principalmente lingüística. 
Por otra parte, es posible distinguir con claridad los momentos históricos de los que el autor da cuenta para analizar la educación en Rapa Nui. Una primera etapa misional marca, según Corvalán (2015), la educación en la isla desde 1862. Esta etapa estuvo caracterizada, según el autor, por "una escolarización incipiente, entendida como evangelización y más propiamente como catecismo y las lenguas tahitiana y francesa, en mixtura con el Rapa Nui, como primeras palabras de transmisión educacional y religiosa cristiana en la isla" (p. 32) Esta etapa se vería interrumpida en 1871 a raíz de "una serie de eventos tormentosos" (p. 33) que no son detallados por el investigador y autor del libro. Una idea es importante destacar en relación a la acción misional educativa en la isla y es que, según Corvalán (2015),

la continuidad desde Eyraud a Pakarati [precursores de la educación en la isla], en total más de 60 años de acción educativa informal bajo el código evangelizador catequístico católico, se dio solo con algunas interrupciones acotadas y sin duda allana el camino para una posterior educación formal la que, junto al nacionalismo chileno, transmitirá contenidos formales y simbólicamente convergentes. (p. 35)

En esta línea, es indudable la adhesión a la tesis de que la educación formal en los inicios fue transformadora no sólo en términos de instalar procesos educativos entre los pascuenses, sino también en cuanto a las transformaciones sociales que produjo en la vida de muchos isleños. Para Corvalán (2015) es incuestionable el hecho de que la función de la escuela, tanto en los inicios como en las etapas de acción estatal directa, consistió en homologar variados códigos culturales de los isleños a los cánones occidentales del continente. En este proceso fueron muy claros los esfuerzos por introducir tanto las creencias religiosas occidentales como el idioma español entre la población Rapa Nui.

En cuanto al material educativo utilizado para la enseñanza en la isla, el primer texto elaborado fue redactado por el subdelegado de Marina Vives Solar. Fue publicado en 1923 y, según el autor, se encontraba en sintonía con los lineamientos establecidos en la ley de instrucción primaria obligatoria promulgada en 1920. En relación al análisis del contenido de estas publicaciones, Corvalán (2015) señala que dan cuenta del etnocentrismo con que desde occidente se asumió la relación pedagógica con Rapa Nui. Como lo señala el autor, luego del análisis de las principales obras, en los textos pueden observarse frases como "hace cinco siglos todos los habitantes de Chile eran indios"; "los indios tenían tanta dificultad para procurarse alimento que siempre andaban con hambre", "no podían hacerse ropa como la que usan los hombres civilizados", "cuando un niño mostraba gusto por la bebida su padre en vez de castigarle, le aplaudía" y "el más infeliz de los actuales pobladores de Chile puede considerarse muy afortunado si compara su situación con la que tenían los indígenas en aquella época tan lejana" (p. 65).

En esta primera parte del libro, es posible constatar también algunas referencias que analizan la enseñanza impartida por los delegados de marina en la isla. Según el autor, el problema de esta educación fue "la falta de idoneidad de sus encargados como lo inadecuado de los métodos pedagógicos y de los contenidos a transmitir" (p. 73). 
Algunas ideas que desde la Antropología Cultural esboza el autor frente a este periodo educativo en la Isla de Pascua están dadas principalmente por que aquella disciplina atraviesa por las marcadas influencias del evolucionismo y esta perspectiva se refleja en el modo en que la intelectualidad concibe la educación, situación que involucra a las acciones pedagógicas en Rapa Nui. En esta línea es posible apreciar a personalidades como Barros Arana o Rodulfo Philippi expresar opiniones con un claro sentido evolucionista, que caracterizaron sus reflexiones. En el caso de este último, tales expresiones se refieren específicamente al caso Rapa Nui.

Una idea si puede quedar en claro para quien lee la primera parte del libro de Javier Corvalán (2015): la concepción de Chile como una nación culturalmente homogénea es también traspasada a la dinámica que asumió la educación en Rapa Nui.

La segunda parte del libro se titula La llegada de la escuela moderna a Rapa Nui, en ella se describe y analiza el período comprendido entre 1960 y 1990. La oferta educativa en este momento se incrementa hasta completar los 8 años de educación formal. A comienzos de la década de 1960 la situación educativa en la isla evidenciaba ciertos cambios con respecto a décadas anteriores y estos pueden resumirse en

primero, que las autoridades continuaban teniendo una buena evaluación del funcionamiento general del establecimiento; segundo, que había conciencia de una precariedad respecto de sus condiciones de funcionamiento; y tercero, que tímidamente se infieren los atisbos de una vinculación educativa entre lo local y lo global desde el momento en que se señala que la educación impartida debe servir para un buen manejo en el continente, pero que al mismo tiempo debe integrar un conjunto de contenidos locales. (p. 111)

Complementando lo anterior, Corvalán (2015) señala que ya hacia 1969 los documentos oficiales de Odeplan mencionan la necesidad de equilibrar los contenidos, rescatando la cultura pascuense e integrándola en las materias trabajadas. También existen menciones sobre la necesidad de formar profesores pascuenses que puedan cumplir con este propósito en la isla.

En este período no existen mayores registros sobre las vivencias cotidianas de niños y niñas continentales y Rapa Nui que hayan ocurrido en la escuela, los primeros hijos e hijas de funcionarios estatales y militares (p. 115). Sería interesante intensificar los esfuerzos por rescatar, desde la oralidad, las características de este encuentro intercultural y analizar de qué manera el contexto socio-histórico influyó en tales características. Lo anterior permitiría comparar la realidad pasada con la presente y describir si han existido continuidades o quiebres en este sentido en Rapa Nui, considerando el componente social e histórico.

Otro elemento importante expuesto en esta parte del libro es que desde la década del 70 comienzan a centrarse las preocupaciones en torno a la lengua Rapa Nui. Odeplan ya consignaba la importancia de enseñar en su lengua a los niños pascuenses en un documento en 1972, y más tarde, en 1977, comenzó el trabajo de la Universidad Católica de Valparaíso y el Instituto Lingüistico de Verano - SIL - de Estados Unidos, con la activa participación del investigador Luis Gómez Macker y los lingüistas Nancy Thielsen y Robert Weber en la isla. 
Es necesario destacar, para este período analizado, que el primer experimento en educación intercultural, diseñado en el período de intervención militar en Chile y publicado en el Diario Oficial en enero de 1976 da cuenta, a juicio del autor, de

un panorama de adecuación o descentralización curricular bastante especial para la époc. [...] Por una parte, se trata de una innovación que con énfasis en la cultura local y específicamente en la lengua rapanui, algo así como un intento de educación intercultural bilingüe cuatro décadas antes de que ella sea parte de las actividades regulares del sistema escolar chileno y además, sin disminuir el tiempo dedicado a otras áreas de aprendizaje, se propone un aumento de las horas lectivas en relación a la educación impartida en el continente. (p. 125)

Paradojalmente, "el 26 de junio de 1977 el Ministerio de Educación promulgó el decreto 500 que modificó el anterior decreto 991 relacionado con el plan de estudios especial para la isla" (p. 128). Con lo anterior, la innovadora propuesta intercultural alcanzó a durar menos de dos años desde su creación.

El año de 1984 marca un hito del período comprendido entre 1960 y 1990, en cuanto se da término a la enseñanza de la lengua rapanui debido a la derogación de los decretos especiales para la Isla de Pascua. Desde este momento se asumen los planes y programas generales del Ministerio de Educación. Lo anterior se ve reforzado también dado el interés de las familias Rapa Nui por escolarización y castellanización de sus hijos en tanto la educación es vista como vehículo de movilidad social.

En la tercera y última parte del libro titulada La complejización de la escolarización en Rapa Nui y la formación de un sistema competitivo en la isla (1990-2014) son analizados los cambios en la concepción de la enseñanza y sus particularidades asociadas a Rapa Nui desde el retorno a la democracia en Chile. Se establecen por parte del autor algunos hitos significativos en términos de la competitividad en la enseñanza, marcada por la instalación de dos colegios privados en los años 2003 y 2006 junto con la división del colegio municipal. Lo anterior determinado contextualmente por la política iniciada en los 1980 con la reforma educacional que impulsaba aquella competencia entre los establecimientos escolares.

La investigación expresada en el libro Educación en Rapa Nui: sociedad y escolarización en Isla de Pascua (1914-2014) da cuenta de una línea histórica completa en cuanto secuencia temporal. Es posible encontrar orden y claridad en la exposición de los contenidos, sin embargo, la indagación podría promover la investigación desde el relato de los actores, en tanto este discurso no aparece expresado de un modo relevante en el libro, quedando implícitamente expresado el desafío de abordar el fenómeno desde los sujetos que vivenciaron los acontecimientos, transformándolos de este modo en la unidad de análisis principal y diversificando las fuentes de información. Lo anterior permitiría contar con otra opción de comprender e interpretar el fenómeno estudiado y posibilitaría una triangulación más amplia de los datos y experiencias, más aún cuando frente a ciertos hechos relatados en el libro aparecen visiones contrapuestas.

En la lectura de la obra reseñada el investigador encontrará datos históricos y estadísticos importantes, y principalmente, podrá comprender la relación que desde el continente se ha sostenido con Rapa Nui en el ámbito educativo junto con el análisis histórico de los factores que han caracterizado tal relación. 


\section{Referencia}

CORVALÁN RODRIGUEZ, Javier. Educación en Rapa Nui: sociedad y escolarización en Isla de Pascua (1914-2014). Santiago de Chile: Universidad Alberto Hurtado, 2015.

ANDRÉS FELIPE BIANCHETTI SAAVEDRA es antropólogo, magíster en Educación y profesor asistente en la Universidad San Sebastián, campus Las Tres Pascualas, Concepción, Chile.

Morada: Lientur 1457 - Concepción - Chile.

E-mail: abianchettis@docente.uss.cl.

Recebido em 9 de junho de 2016

Aceito em 2 de agosto de 2016. 